outside the collision avoidance rules have to be sought. Traffic separation schemes go a long way and further measures may eventually be called for, such as the application of queuing theory, scheduling systems in narrow waters, \&c. This suggests industrial integration far beyond the area of maritime transport.

An appreciation of the build up of events preceding a close quarters situation is encouraged by the simulation exercises used in navigation schools. It would be enhanced by the personal involvement resulting from a study of near misses which had actually been experienced. No improvement short of complete and universal automation can be of much avail until the collision avoidance instinct within each officer has been developed to the full.

\title{
The Operation of Light Aircraft
}

An informal discussion on the operation of light aircraft took place at a meeting of the Technical Committee of the Institute in London on 21 April 1976. A personal view expressed by Mr. F. S. Stringer and comments by Mr. C. Powell and Professor A. N. Black are printed below.

\section{F. S. Stringer}

RECENT technical press disclosures have highlighted C.A.A. concern that the London Air Traffic Zone is being entered by unauthorized light aircraft, the infringements varying from minor boundary incursions to major crossings of the area. The pilots involved vary in experience, and in most if not all cases have been flying under VFR rules and out of radio contact with Air Traffic Control. It seems very probable that these pilots are lost, or at least very unsure of their position; the topographical relation of charts to places on the ground is becoming increasingly difficult to interpret due to the complexity of built-up areas, especially around the T.M.A.s. Not all aircraft carry VOR or ADF and the map and DR are the main navigational aids; some pilots are reluctant to call for radar assistance for a variety of reasons.

A ground positioned strobo-flash can be seen from the air in marginal visibility, as witnessed on many general aviation aircraft already fitted with these lights for normal identification purposes. A small number of such lights positioned strategically in and around the T.M.A. could be used as a safety check by pilots flying VFR to ensure that they do not stray into the zone. The number of beacons could be limited and placed at points of particular traffic density, such as the southern boundary extremities of the London Zone. Pilots could ensure that in VFR the beacon was seen, and identified as positioned correctly relative to their flight path, before turning on to a new heading to complete a track around the prohibited zone. The beacons would be no more expensive than those now fitted to aircraft and no special flashing code would be necessary, though this would probably be advantageous. The beacons could be fitted on telegraph poles and shielded from ground observation to prevent annoyance to the general public. Since the real estate required could be minimal and probably rented, say on the roof of a building, one difficulty in providing ground based radio aids to navigation should not be experienced. 
In recent years there has been a significant increase in the numbers of general aviation aircraft within the U.K. though less than elsewhere in Europe, especially France and Germany. In addition there has been an increase in the navigational and air traffic control over the United Kingdom and the remainder of Europe. It is therefore essential for safety that pilots receive adequate instruction not only in basic navigation but navigation within the air traffic environment, coupled with the associated communication system operation skills. The existing I.M.C. rating provides a private pilot with instrument flying capability sufficient to permit him to fly in marginal weather and to extract himself in circumstances where deteriorating conditions could otherwise prove hazardous. There is however a need for constant updating on procedural air traffic and navigational arrangements and facilities, and interpretation of any new regulations. Though a limited number of centres provide excellent instructional courses in these subjects there is a scarcity of informed assistance generally. Learned societies might encourage the presentation of such information at lecture series and seminars, both to encourage interest and to permit ready access by private pilots to an official interpretation of what the private aviator may and may not do.

Radio aids to the navigation of light aircraft, particularly in the United Kingdom, comprise VOR, ADF and radio communication for contact with ground radar services provided by Air Traffic Control. The VOR, and to a lesser extent ADF, provide a simple and effective means of navigation by checking gross errors in dead reckoning. A major share of the equipment fit seems to be of U.S. origin, generally built to ARINC standards; the systems are usually reliable but costly. The installation of such systems retrospectively can also be costly and requires cockpit panel space which is often not available.

Large scale and medium scale integrated solid state component technology could make its application felt in the general aviation field. Though the U.K. market is not large in itself the potential overseas market is huge, as expressed in a recent symposium arranged by the Royal Aeronautical Society. Two factors appear to deter British industry from entering the field, the capital cost of unit development and the U.S. lead in the existing market. Encouragement for market research by learned societies may be worth consideration before a more determined effort to enter the field is attempted. The provision of small and readily installed solid state communications and VOR equipment would appear to be the most appropriate item for initial consideration. To make a significant impact, new design concepts would be needed to ensure a marked decrease in cost coupled with high reliability. If a study revealed this to be a worthwhile subject for practical application the overall improvement to navigation generally, with consequent increase in safety to all air users, would be ample reward.

The appearance of the Stogmuller type of radio navigation chart for use with VOR and DME in aircraft has prompted studies at Cranfield and in the U.S.A. to examine its potential in the field of general aviation. The technique, which has been known for some years and applied in some Decca equipment, involves in the Stogmuller case the transfer of polar coordinates to a cartesian grid. This allows easy operation of VOR or DME by a pilot since interpretation of position is more readily achieved, particularly if the intended waypoints are plotted on the chart before flight. The topographical grids are of course distorted. The method of operation needs very little instruction and it has been found to provide a high order of navigational accuracy, dependent on the basic accuracy of the navigational system and its operation. 
One school of thought suggests that this very cheap and simple device could reduce the required standard of continuation training in DR navigation and map reading. This is debatable but there are several attractive features of the Stogmuller charts, not the least being the easy identification of gross errors. The technique has been extended to approach and landing charts and further developments in automatic computation and display are worth study. Transfer of polar to cartesian coordinates in a solid state micro-processor and the presentation of the intersection point of pairs of lines on a solid state display might reduce the workload for less practiced pilots. The immediate knowledge of position provided, both in the air and at A.T.C. Centres, would benefit air safety.

\section{Claud Powell}

THE possible manufacture of microminiature navigation equipment for light aircraft is engaging the attention of manufacturers in Europe as well as in the U.S.A., but those in the U.K. face the formidable handicap of the size of the ready-made market enjoyed by the American manufacturers. The low prices for which pocket calculators can now be purchased are simply the result of the vast size of the domestic market and unfortunately do not imply any possibility of correspondingly cheap airborne equipment.

On the subject of Stogmuller charts, similar 'inverse lattices' were used with Loran during the War, and were adopted by Decca for airborne displays. Decca soon found however that the gross distortion resulting from a simple representation of the position lines as a rectilinear grid was quite unacceptable, except in areas where the angle of cut was large, and that even the more moderate distortion gained by modifying the lattice on the basis of sum and difference patterns remained unacceptable to many users. What is gained by invoking the gross distortion, particularly near a VOR, of a Stogmuller chart? Granting the need for a separate plotting chart containing minimal topographic detail, why not display the VOR radials and DME circles in their true form on such a chart so that it becomes compatible with a conventional map?

\section{Professor A. N. Black}

THE proposed boundary marker beacons should have distinctive characteristics for positive identification of one from another. A pilot who is uncertain of his position might well see a beacon as the one certain clue to his location and if he makes a wrong identification this will certainly make him reluctant to accept other clues which would correct him. Amateurs are probably particularly prone to this psychological block, since they lack the continuous day-to-day experience of professionals. 